\title{
Channelling bias and the incidence of gastrointestinal haemorrhage in users of meloxicam, coxibs, and older, non-specific non-steroidal anti-inflammatory drugs
}

\author{
T M MacDonald, S V Morant, J L Goldstein, T A Burke, D Pettitt
}

Gut 2003;52:1265-1270

See end of article for authors' affiliations

Correspondence to: Professor T M MacDonald Medicines Monitoring Unit Department of Clinical Pharmacology and Therapeutics, University of Dundee, Ninewells Hospital and Medical School, Dundee DD1 9SY, UK

Tom@memo.dundee.ac.uk

Accepted for publication 30 April 2003
Background: Although clinical trial results suggest that meloxicam has less gastrointestinal toxicity than most other non-steroidal anti-inflammatory drugs (NSAIDs), in practice it has been associated with a large number of yellow card reports of gastrointestinal complications.

Aims: To estimate whether meloxicam and the coxibs, rofecoxib and celecoxib, have been channelled towards high risk patients, and to estimate the risk of hospitalisation for gastrointestinal haemorrhage associated with the use of these drugs, allowing for the effects of channelling.

Patients: Using the UK General Practice Research Database, this study included 7. 1 thousand patient years (tpy) exposure to meloxicam, 1.6 tpy exposure to coxibs, and 628 tpy exposure to older nonspecific NSAIDs.

Methods: Cohort study of patients who received a prescription for an NSAID between June 1987 and January 2001. Exposure to newer NSAIDs (meloxicam, rofecoxib, celecoxib) and to older non-specific NSAIDs was identified. Channelling was assessed on factors including: demographic variables; diagnosis of arthritis; history of NSAID use or gastrointestinal events, including gastrointestinal haemorrhage; and use of ulcer healing drugs.

Results: Most risk factors for gastrointestinal haemorrhage were more prevalent among patients prescribed the newer NSAIDs. Adjusting for these risk factors reduced the relative risks of gastrointestinal haemorrhage on meloxicam and coxibs versus older non-specific NSAIDs to 0.84 (95\% confidence interval $0.60,1.17)$ and $0.36(0.14,0.97)$, respectively.

Conclusions: Channelling towards high risk gastrointestinal patients occurred in the prescribing of newer NSAIDs. After attempting to correct for channelling bias, coxib exposure, but not meloxicam exposure, was associated with a significantly lower risk of gastrointestinal haemorrhage than older non-specific NSAID exposure.
M eloxicam was introduced in the UK for the short term treatment of osteoarthritis (OA) and the long term treatment of rheumatoid arthritis (RA) in 1996. Clinical trial results have suggested that the gastrointestinal toxicity of meloxicam was less than that of most other non-steroidal anti-inflammatory drugs (NSAIDs), ${ }^{1}$ but in practice it has been associated with a large number of yellow card reports of gastrointestinal complications. ${ }^{2}$ This may be due to the fact that meloxicam was marketed as a "safer" NSAID. As a result, in clinical practice it may be channelled towards patients at high risk of gastrointestinal haemorrhage. Such channelling towards high risk patients, together with a reporting bias stimulated by its status as a new drug, may account for the high rate of reporting of upper gastrointestinal complications that occurred in patients who received meloxicam. ${ }^{3}$

The coxibs, including celecoxib and rofecoxib, are a new class of NSAIDs. These drugs appear to have significant upper gastrointestinal safety advantages over non-specific NSAIDs, which inhibit both the cyclooxygenase (COX)-1 and COX-2 enzymes. ${ }^{4-10}$ These drugs may also be channelled towards use in patients at high risk of gastrointestinal haemorrhage, and upper gastrointestinal event rates of patients taking coxibs may compare unfavourably with those taking conventional NSAIDs as a result of such channelling.

The objectives of this study were to determine whether channelling occurred in the prescribing of meloxicam and coxibs in the UK primary care setting, and to estimate the extent of such channelling. In addition, the risk of hospitalisation for upper gastrointestinal haemorrhage associated with the use of these newer drugs, relative to older non-specific NSAIDs, was estimated, allowing for the effects of channelling.

\section{METHODS}

\section{Source population}

The study was carried out in the setting of UK primary care, using data from practices that participated in the General Practice Research Database (GPRD). This database has been described extensively. ${ }^{11}$ The GPRD contains details of patients' demographics, medical diagnosis, referrals to consultants and hospitals, and prescriptions. The accuracy and completeness of the data in the GPRD have been validated in previous studies. ${ }^{12}$ The source population data were available for 686 practices between June 1987 and January 2001.

\section{Study population}

Study practices were defined as those that prescribed any meloxicam or coxibs in the period during which they met the audit standard for research (up to standard (UTS) period). The study population consisted of all patients in these practices. The study period started with the first use of meloxicam or a

Abbreviations: NSAIDs, non-steroidal anti-inflammatory drugs; COX, cyclooxygenase; GPRD, General Practice Research Database; UTS, up to standard; OA, osteoarthritis; RA, rheumatoid arthritis; CCB, calcium channel blockers; ptpy, per thousand patient years; GP, general practitioner; $R R$, relative risk. 
Table 1 NSAID prescribing rates and gastrointestinal haemorrhage rates in all patients in the study practices and in other practices in the GPRD

\begin{tabular}{lcc}
\hline & Study practices & Other practices \\
\hline No of practices & 369 & 317 \\
Patient years (thousands) & 22064 & 8418 \\
NSAID prescriptions (thousands) & 10128 & 3249 \\
NSAID prescriptions ptpy & 459 & 386 \\
Gastrointestinal haemorrhages (incident cases) & 26068 & 10054 \\
Gastrointestinal haemorrhages ptpy & 1.18 & 1.19
\end{tabular}

NSAIDs, non-steroidal anti-inflammatory drugs; GPRD, General Practice Research Database; ptpy, per

thousand patient years.

coxib in each practice, within its UTS period. Study subjects were those patients in the study population who received any NSAID during the study period. Thus comparisons between newer NSAIDs and older non-specific NSAIDs were made within periods of time when practices made both types of drug available.

\section{Study design}

This was a cohort study of NSAID users. A single cohort was defined, consisting of all the individuals in each practice who received an NSAID prescription. Most users of meloxicam or coxibs were also exposed to older non-specific NSAIDs at different times during the study period.

\section{Exposure}

Periods of exposure and non-exposure to meloxicam, coxibs, and older non-specific NSAIDs were identified for every patient. Any prescriptions for which an exposure period could not be calculated were assumed to provide 28 days exposure. Any prescription that was issued less than 15 days after the end of the exposure period implied by the previous prescription was assumed to provide continuous exposure.

The first day of each period of continuous exposure to an NSAID defined a set of index dates for each patient. Periods of non-exposure were also identified, and those prior to first use of an NSAID within the study period were distinguished from subsequent periods of non-exposure. Periods of exposure to aspirin alone were excluded from the analyses, but periods of simultaneous exposure to aspirin and an NSAID were assigned to the NSAID.

\section{Outcome events}

The outcome of interest was gastrointestinal haemorrhage, melaena, or haematemesis (see appendix for the OXMIS and Read codes for these parameters).

\section{Control of bias}

The following factors were evaluated on every index date for each patient: sex; age; any diagnosis of OA; any diagnosis of $\mathrm{RA}$; recent diagnosis of sprain or strain; recent diagnosis of back pain, history of NSAID use; history of gastrointestinal haemorrhage, recent gastrointestinal endoscopy; recent/any history of gastrointestinal events (erosions, ulcers or bleeding); recent/any history of dyspepsia (including abdominal pain); recent/any prescriptions for ulcer healing drugs; recent prescriptions for nitrates, calcium channel blockers (CCB), or antacids and other drugs used to treat dyspepsia; alcohol consumption; and tobacco consumption. Channelling bias due to these factors may confound comparisons between newer NSAIDs and older non-specific NSAIDs. An event was defined as "recent" if it occurred within 30 days prior to the index date. In most cases a prescription within 30 days of an index date implied concomitant exposure to that drug on the index date.

Because the number of factors was large, matching on all of them within individual general practitioner (GP) practices was not possible. Instead, we included each of them as a covariate in the statistical models. We also used a propensity score calculated using the same factors. Rosenbaum and Rubin ${ }^{13}$ showed that, under appropriate conditions, stratifying on the propensity score can achieve balance within strata simultaneously for a large number of factors.

The extent of channelling on any of these factors may vary between practices, depending on individual GP's perceptions of the risks associated with them. Therefore, we tested for channelling effects on each factor averaged across the practices with a random effects model, using the methods described by Whitehead and Whitehead. ${ }^{14}$

\section{Outcome rates}

The numbers of outcome events and the exposure times were aggregated for every combination of potential risk factors in the analysis. For the purposes of these analyses, age was categorised by decade and a maximum of five levels was used to categorise other continuous risk factors. The numbers of events were modelled as a Poisson variable with log (exposure time) as an offset variable for different durations of therapy. The statistical models included the individual risk factors as covariates, the propensity score categorised by quintiles of the distribution for newer NSAIDs, or both. Unless otherwise stated, we present relative risks and their associated confidence intervals and $\mathrm{p}$ values from the model that did not use the propensity score.

\section{RESULTS}

NSAID prescribing rates and gastrointestinal haemorrhage rates in study practices (those prescribing newer NSAIDs, $\mathrm{n}=369$ ) were similar to those in practices from the GPRD that were not included in this study (those prescribing only older non-specific NSAIDs, $n=317$ ) (table 1 ). The rates of gastrointestinal haemorrhages/thousand patient years (ptpy) were 1.18 for the study practices compared with 1.19 ptpy for other practices.

\section{Events}

The total exposure time to newer NSAIDs and older non-specific NSAIDs during the UTS periods in the 369 practices that prescribed meloxicam or coxibs is given in table 2 . A total of 1.38 million patients (all NSAID users) were included in the analysis. The total number of upper gastrointestinal haemorrhages in the study population was 8526 , a rate of 1.32 ptpy. The event rate during meloxicam exposure was 5.1 ptpy; the event rate during coxib exposure was 2.6 ptpy; and it was 4.6 ptpy for older non-specific NSAIDs (excluding aspirin) (table 2). Some of the difference in these event rates may be due to channelling effects, which result in different risk profiles in patients receiving different types of NSAID.

\section{Channelling}

Table 3 summarises the channelling bias for individual risk factors between newer NSAIDs and older non-specific NSAIDs during the study period. Patients who received newer NSAIDs 
Table 2 Exposure to newer NSAIDs and older non-specific NSAIDs during the study period in study subjects from 369 study practices

\begin{tabular}{lccl}
\hline Period & Patient years* ('000s) & No of events & Event rate (ptpy) \\
\hline Total & 6453 & 8526 & 1.32 \\
Before first exposure & 3136 & 2397 & 0.8 \\
Meloxicamt & 7.1 & 36 & 5.1 \\
Coxibs & 1.6 & 4 & 2.6 \\
Older non-specific NSAIDsł & 628 & 2875 & 4.6 \\
Post exposure & 2680 & 3214 & 1.2 \\
\hline \multirow{2}{*}{ NSAIDs, non-steroidal anti-inflammatory drugs; ptpy, per thousand patient years. } \\
*A total of 1 383 744 patients, all NSAID users, were included in the analysis. \\
†56\% of exposure at 7.5 mg/day, 38\% at 15 mg/day. \\
†Excludes exposure to aspirin alone.
\end{tabular}

\begin{tabular}{|c|c|c|c|}
\hline \multirow[b]{2}{*}{ Risk factor } & \multicolumn{2}{|c|}{ Newer NSAIDs } & \multirow{2}{*}{$\begin{array}{l}\text { Older non-specific } \\
\text { NSAIDs }\end{array}$} \\
\hline & Coxibs & Meloxicam & \\
\hline Age (y) & 63.9 & 62.1 & 59.8 \\
\hline Male (\%) & 29.2 & 31.9 & 38.3 \\
\hline Moderate or heavy drinker (\%) & 0.8 & 1.1 & 3.2 \\
\hline Moderate or heavy smoker (\%) & 1.1 & 2.4 & 6.8 \\
\hline Recent endoscopy $(\%)$ & 0.1 & 0.2 & 0.0 \\
\hline Recently diagnosed gastric event $(\%)$ & 1.8 & 1.2 & 0.6 \\
\hline History of $\mathrm{H}_{2}$ antagonist use (\%) & 42.4 & 34.9 & 14.6 \\
\hline History of PPI use (\%) & 35.9 & 23.5 & 4.9 \\
\hline History of misoprostol use (\%) & 3.4 & 3.3 & 1.9 \\
\hline Recently diagnosed dyspepsia (\%) & 0.3 & 0.2 & 0.1 \\
\hline Recent use of antacids (\%) & 6.9 & 5.6 & 3.9 \\
\hline Recent use of UHDs (\%) & 23.4 & 19.4 & 8.1 \\
\hline$\%$ of prior 6 months exposed to UHDs & 2.1 & 2.3 & 1.9 \\
\hline Any history of dyspepsia (\%) & 17.5 & 12.7 & 6.1 \\
\hline Any history of gastrointestinal events (\%) & 35.2 & 25.8 & 11.7 \\
\hline Recent use of local anaesthetics (\%) & 6.9 & 5.6 & 3.9 \\
\hline OA patients $(\%)$ & 34.3 & 30.5 & 19.8 \\
\hline Recently diagnosed OA (\%) & 9.5 & 5.8 & 2.5 \\
\hline RA patients (\%) & 10.4 & 11.7 & 7.1 \\
\hline Recently diagnosed RA (\%) & 2.1 & 2.3 & 0.9 \\
\hline Exposure initiated after recent back pain (\%) & 4.0 & 5.7 & 5.1 \\
\hline Exposure initiated after a recent sprain or strain (\%) & 0.6 & 0.6 & 1.3 \\
\hline Repeat NSAID users (\%) & 93.5 & 92.6 & 81.0 \\
\hline$\%$ of prior 6 months exposed to NSAIDs & 2.1 & 2.3 & 1.9 \\
\hline No of GP contacts in prior 6 months & 1.8 & 1.6 & 1.1 \\
\hline Recent use of nitrates and CCBs (\%) & 12.4 & 11.8 & 9.1 \\
\hline Recent use of corticosteroids (\%) & 4.6 & 4.8 & 2.5 \\
\hline Recent use of anticoagulants (\%) & 0.7 & 0.4 & 0.3 \\
\hline \multicolumn{4}{|c|}{$\begin{array}{l}\text { NSAIDs, non-steroidal anti-inflammatory drugs; PPI, proton pump inhibitor; UHDs, ulcer healing drugs; OA, } \\
\text { osteoarthritis; RA, rheumatoid arthritis; CCB, calcium channel blockers; GP, general practitioner. } \\
\text { p }<0.001 \text { for channelling bias (newer } v \text { older NSAIDs) averaged over all practices for all factors in a random } \\
\text { effects models. } p<0.001 \text { for heterogeneity between GP practices for all factors except recent history of sprai } \\
\text { or strain. }\end{array}$} \\
\hline
\end{tabular}

were on average 2.6 years older than those who received older non-specific NSAIDs. Males and patients known to be moderate or heavy drinkers or smokers were proportionately less likely to have received newer NSAIDs.

All of the factors indicating gastrointestinal symptoms or a perceived risk of gastrointestinal complications were more prevalent in patients who received newer NSAIDs. In particular, histories of dyspepsia and gastric events and the use of ulcer healing drugs, recently or at any time prior to the index date, were at least twice as common in patients who received newer NSAIDs compared with those who received older nonspecific NSAIDs. They were also more common in coxib users than meloxicam users.

Patients with a recorded diagnosis of OA or RA, either in the last 30 days or at any time, were more likely than other patients to have received newer NSAIDs, whereas those recently diagnosed with a sprain or a strain were less likely to have received them. Repeat NSAID users were more likely to have received newer NSAIDs, and patients who received newer NSAIDs had more NSAID exposure in the previous six months.

Patients who received newer NSAIDs had more contacts with their GP in the six months prior to their index dates, and more prescriptions for nitrates, CCBs, corticosteroids, and anticoagulants compared with older non-specific NSAID users.

There was heterogeneity between GP practices in the extent to which they channelled patients, which was significant $(p<0.001)$ for each of the factors in table 3, except recent history of sprain or strain. Nevertheless, tests for channelling bias averaged over all practices were also significant $(p<0.001)$ in a random effects model. Thus despite differences between GPs in their perception of the risk posed by various factors, there was still clear evidence that overall the risk profiles of patients who received newer and older NSAIDs were systematically different. 
Table 4 Relative risks of gastrointestinal haemorrhage for newer NSAIDs versus older non-specific NSAIDs

\begin{tabular}{|c|c|c|c|c|}
\hline & \multicolumn{2}{|l|}{ Meloxicam } & \multicolumn{2}{|l|}{ Coxibs } \\
\hline & Relative risk $(95 \% \mathrm{CI})$ & $\mathrm{p}$ Value & Relative risk $(95 \% \mathrm{CI})$ & $\mathrm{p}$ Value \\
\hline Unadjusted & $1.11(0.80,1.50)$ & 0.532 & $0.56(0.21,1.50)$ & 0.248 \\
\hline \multicolumn{5}{|l|}{ Adjusted* } \\
\hline Covariates & $0.84(0.60,1.17)$ & 0.303 & $0.36(0.14,0.97)$ & 0.043 \\
\hline Propensity score $†$ & $0.84(0.60,1.18)$ & 0.307 & $0.40(0.15,1.06)$ & 0.066 \\
\hline Both & $0.84(0.60,1.18)$ & 0.418 & $0.36(0.13,0.97)$ & 0.043 \\
\hline
\end{tabular}

NSAIDs, non-steroidal anti-inflammatory drugs.

${ }^{*}$ Adjusted for sex, age, any diagnosis of osteoarthritis, any diagnosis of rheumatoid arthritis, recent

diagnosis of sprain or strain, recent diagnosis of back pain, history of NSAID use, history of gastrointestinal

haemorrhage, recent gastrointestinal endoscopy, any and recent history of gastrointestinal events (erosions,

ulcers or bleeding), any and recent history of dyspepsia (including abdominal pain), any and recent

prescriptions for ulcer healing drugs, recent prescriptions for nitrates, calcium channel blockers or antacids,

alcohol consumption, and tobacco consumption.

tStratified by quintiles of the propensity score distribution for newer NSAIDs.

\section{Risk factors for gastrointestinal haemorrhage} Demographic factors

Age was a major risk factor $(\mathrm{p}<0.001)$, with risks rising by a factor of $6.28(5.34,7.38)$ between the ages of 50 and 90 years. We also found a marked sex difference $(\mathrm{p}<0.001)$, the relative risk ( $R R)$ for males versus females being 1.40 (1.30, 1.51). There was evidence that heavy smokers were at excess risk compared with non-smokers (RR 1.41 ( 1.08, 1.84), p<0.001). The RR in heavy drinkers compared with non-drinkers was $1.31(0.67,2.53)$.

\section{Gastrointestinal history}

Factors associated with a significant increase in the risk of gastrointestinal haemorrhage were a recently recorded gastric event (RR $1.70(1.23,2.36) ; p=0.001)$, any previous prescription for $\mathrm{H}_{2}$ antagonists (RR 1.24 (1.10, 1.40); $\mathrm{p}<0.001$ ), and a recent prescription for antacids ( $R R \quad 1.20$ (1.04, 1.39); $\mathrm{p}<0.001)$. As this study included only the first occurrence of gastrointestinal haemorrhage in each patient, it provided no information on the risks associated with a prior history of the outcome event.

\section{Indication}

There was no evidence that the indication for which NSAIDs were prescribed was associated with the risk of gastrointestinal haemorrhage. Users who had previously been exposed to NSAIDs were at less risk than first time users (RR $0.51(0.45,0.57) ; \mathrm{p}<0.001)$. The risk of gastrointestinal haemorrhage also diminished with increasing exposure to NSAIDs in the prior six months $(p<0.001)$. These findings probably reflect a reluctance to prescribe NSAIDs to patients at higher risk of gastrointestinal side effects.

\section{Comorbidities}

There was a high correlation between the number of GP visits in the six months prior to a period of NSAID exposure (a general marker of comorbidity) and the risk of gastrointestinal haemorrhage during that period of exposure $(p<0.001)$. The RR for patients with five or more visits was $4.86(4.03,5.87)$ relative to those with none. Other markers of comorbidity were also risk factors. The RRs for recent use of anticoagulants and corticosteroids were, respectively, $2.79(2.09,3.73)$ and $1.68(1.44,1.97)$. In contrast, use of nitrates and CCBs did not appear to be risk factors for gastrointestinal haemorrhage.

\section{Relative risks for newer NSAIDs versus older non-specific NSAIDs}

The unadjusted and risk adjusted RRs of gastrointestinal haemorrhage for meloxicam and coxibs compared with older non-specific NSAIDs are given in table 4 . There was no significant difference in the RRs for meloxicam compared with older non-specific NSAIDs. For coxibs, however, the risk adjusted RR was significantly lower $(\mathrm{p}=0.043)$.

Analyses stratified by propensity score, with or without individual covariate adjustments, yielded identical estimates of the RR for meloxicam (0.84). The RR for coxibs was slightly higher $\mathrm{RR}(0.40)$ in an analysis stratified by propensity score alone.

\section{DISCUSSION}

In this study, we observed an approximately sixfold increase in the rate of gastrointestinal haemorrhage during exposure to older non-specific NSAIDs relative to the pre-exposure periods. This is consistent with previous studies which reported an eightfold increase in hospitalisation rates for complicated gastrointestinal events and a fourfold increase in hospitalisation for any reported gastrointestinal event. ${ }^{15}$ Rates of gastrointestinal haemorrhage following NSAID exposure were about $50 \%$ higher than they were before first exposure, a pattern that has also been found previously with broader categories of gastrointestinal events associated with hospitalisations. ${ }^{6}$

We found clear evidence that meloxicam and the coxibs, rofecoxib and celecoxib, were used in patients with different characteristics from users of older non-specific NSAIDs and that channelling of high risk patients to the newer NSAIDs occurred. By comparison, the risk profiles of coxib and meloxicam users were similar, although there was evidence that some risk factors were more prevalent among coxib users. We also found that the unadjusted gastrointestinal haemorrhage rates were higher in patients receiving the newer NSAIDs. We attempted to correct for the impact of channelling on gastrointestinal haemorrhage rates by covariate adjustment. Observed gastrointestinal haemorrhage rates on the newer NSAIDs were adjusted downwards to account for the channelling of older patients and those with a gastrointestinal history onto these agents. An upward adjustment was required to correct for channelling on sex. Other factors gave rise to small adjustments, either because they were not risk factors or because they were not associated with channelling. For example, there appeared to be no difference in gastrointestinal event rates between arthritis patients and those with musculoskeletal pain. Conversely, the recent use of anticoagulants was a major risk factor, but these drugs were used to a similar extent among patients receiving newer NSAIDs and older non-specific NSAIDs. Overall, covariate analyses reduced the risk of gastrointestinal haemorrhage on meloxicam from an unadjusted rate of 1.11 relative to that for older non-specific NSAIDs to 0.84 , and reduced the unadjusted rate on coxibs from 0.56 to 0.36 . These findings are in agreement with two recently published studies. ${ }^{16}{ }^{17} \mathrm{~A}$ meta analysis of randomised trials comparing celecoxib with traditional 
NSAIDs or placebo showed a lower incidence of upper gastrointestinal complications with celecoxib. ${ }^{16}$ An observational cohort study found a significantly lower risk of short term upper gastrointestinal haemorrhage for the coxibs compared with older non-specific NSAIDs.

We also attempted to adjust for channelling bias by stratifying on a propensity score. The estimate of relative risk for meloxicam in this analysis was identical $(0.84)$. For the coxibs it was slightly higher $(0.40 v 0.36)$, but covariate adjustments within the strata of the propensity score distribution reduced it $(0.36)$.

As with any observational study, unrecorded risk factors may bias these results. Other studies ${ }^{6}$ have shown that social deprivation score is a risk factor for gastrointestinal complications requiring hospitalisation. It may also influence the likelihood of receiving the newer NSAIDs. It was not possible to establish social deprivation scores in our study. Previous studies have also shown that the likelihood of receiving rofecoxib and celecoxib was related to a chronic disease score in arthritis patients, ${ }^{18}$ and the same may be true of meloxicam. We were not able to calculate a similar score in the present study, although the amount of NSAID exposure in the six months prior to an index date and the use of corticosteroids may be related to severity of disease.

A strength of this study is that it used data that were recorded by GPs as part of their normal practise, and it therefore reflects the standard of care in a primary care setting in the UK. A weakness of this and other studies that rely on such databases is the lack of a reliable denominator, due to uncertainty over the status of patients who have no contact with the practices at which they are registered. We attempted to limit the effects of this uncertainty by using exposure times to prescribed NSAIDs as our denominator, so that only patients who contacted their GPs to obtain prescriptions were included.

Another weakness is that we relied on prescription data and assumed that there was full compliance with prescribed medication. We could not assess compliance in this study but it has been reported elsewhere that $10-15 \%$ of prescriptions are not dispensed. ${ }^{19}$ Compliance might be expected to be higher on the coxibs because lower rates of intolerance related withdrawals have been reported in clinical trials. ${ }^{8}{ }^{10}$ If this is the case, our study underestimates gastrointestinal haemorrhage rates during exposure to older non-specific NSAIDs to a greater extent than the rates on newer agents.

This analysis included patients who were exposed to aspirin at the same time as an NSAID but we did not attempt to establish the risks attributable to aspirin, nor whether risks were modified by concomitant aspirin exposure.

Outcome events in this study were not verified and there may be some overcounting, although previous studies using the same database suggest that the number of false positive diagnoses of gastrointestinal haemorrhage is likely to be small. ${ }^{11}$ Conversely, some outcome events may be missing due to hospitalisations not being recorded by the GP practice. It is unlikely that either of these recording errors would have been systematically biased in favour of or against a particular NSAID class.

This study shows that when the newer NSAIDs were introduced they were channelled to particular groups of patients. In situations where indication or contraindication biases exist, and complex channelling effects can be expected, only randomised trials can be relied upon to provide unbiased treatment comparisons. Conventional randomised controlled clinical trials are expensive, involve relatively small numbers of patients, and the potential to generalise their results can be limited. A study design which, ethical considerations permitting, allowed drug allocation to be randomised in an otherwise normal clinical setting, and which relied upon the routine collection of primary and secondary health care records, could overcome the size limitations and atypical settings of conventional clinical trials. It would also avoid the channelling bias that may, in some cases, make it impossible to interpret the results of purely observational studies.

In conclusion, this cohort study using the General Practice Research Database showed that channelling towards high risk gastrointestinal patients did occur in the prescribing of meloxicam and the coxibs, celecoxib and rofecoxib, in a UK primary care setting. After attempting to correct for channelling bias, coxib exposure, but not meloxicam exposure, was associated with a significantly lower risk of gastrointestinal haemorrhage than conventional NSAID exposure.

\section{ACKNOWLEDGEMENTS}

This study was funded by Pfizer Inc., New York, USA

\section{APPENDIX}

Table Al shows the OXMIS and Read codes for outcome events.

Table A1 OXMIS and Read codes for outcome events

\begin{tabular}{|c|c|c|}
\hline Coding system & Code & Translation of the code \\
\hline \multirow[t]{8}{*}{ OXMIS } & $5339 \mathrm{DB}$ & Duodenal ulcer bleeding \\
\hline & $569 \mathrm{M}$ & Gastrointestinal bleeding \\
\hline & $569 \mathrm{ME}$ & Upper gastrointestinal haemorrhage \\
\hline & $569 \mathrm{MH}$ & Intestinal haemorrhage \\
\hline & $569 \mathrm{Ml}$ & Haemorrhage gastrointestinal \\
\hline & 7845 & Haematemesis \\
\hline & & Melaena \\
\hline & 19E4.12 & $\mathrm{C} / \mathrm{O}-$ melaena \\
\hline \multirow[t]{28}{*}{ Read } & 4737.11 & Melaena-O/E of faeces \\
\hline & 7619100 & $\begin{array}{l}\text { Gastrotomy and ligation of bleeding } \\
\text { point of stomach }\end{array}$ \\
\hline & $\mathrm{J} 110100$ & Acute gastric ulcer with haemorrhage \\
\hline & $\mathrm{J} 110111$ & Bleeding acute gastric ulcer \\
\hline & $\mathrm{J} 110300$ & $\begin{array}{l}\text { Acute gastric ulcer with haemorrhage } \\
\text { and perforation }\end{array}$ \\
\hline & $\mathrm{J} 111100$ & $\begin{array}{l}\text { Chronic gastric ulcer with } \\
\text { haemorrhage }\end{array}$ \\
\hline & J1ו1ו1ו1 & Bleeding chronic gastric ulcer \\
\hline & $J 111300$ & $\begin{array}{l}\text { Chronic gastric ulcer with } \\
\text { haemorrhage and perforation }\end{array}$ \\
\hline & J11y100 & $\begin{array}{l}\text { Unspecified gastric ulcer with } \\
\text { haemorrhage }\end{array}$ \\
\hline & J11y300 & $\begin{array}{l}\text { Unspecified gastric ulcer with } \\
\text { haemorrhage and perforation }\end{array}$ \\
\hline & J1 lyy00 & $\begin{array}{l}\text { Unspecified gastric ulcer; unspecified } \\
\text { haemorrhage and/or perforation }\end{array}$ \\
\hline & $\mathrm{J} 120100$ & $\begin{array}{l}\text { Acute duodenal ulcer with } \\
\text { haemorrhage }\end{array}$ \\
\hline & $J 120300$ & $\begin{array}{l}\text { Acute duodenal ulcer with } \\
\text { haemorrhage and perforation }\end{array}$ \\
\hline & $\mathrm{J} 121100$ & $\begin{array}{l}\text { Chronic duodenal ulcer with } \\
\text { haemorrhage }\end{array}$ \\
\hline & $J 121111$ & Bleeding chronic duodenal ulcer \\
\hline & $J 121300$ & $\begin{array}{l}\text { Chronic duodenal ulcer with } \\
\text { heemorrhage and perforation }\end{array}$ \\
\hline & $J 12 y 100$ & $\begin{array}{l}\text { Unspecified duodenal ulcer with } \\
\text { haemorrhage }\end{array}$ \\
\hline & $\mathrm{J} 12 \mathrm{y} 300$ & $\begin{array}{l}\text { Unspecified duodenal ulcer with } \\
\text { haemorrhage and perforation }\end{array}$ \\
\hline & $\mathrm{J} 12 \mathrm{yy} 00$ & $\begin{array}{l}\text { Unspecified duodenal ulcer; } \\
\text { unspecified haemorrhage and/or } \\
\text { perforation }\end{array}$ \\
\hline & $J 68 . .00$ & Gastrointestinal haemorrhage \\
\hline & 1680.00 & Haematemesis \\
\hline & 1681.00 & Melaena \\
\hline & $168 z .00$ & $\begin{array}{l}\text { Gastrointestinal haemorrhage } \\
\text { unspecified }\end{array}$ \\
\hline & J68z.11 & GIB-gastrointestinal bleeding \\
\hline & $J 68 z 000$ & Gastric haemorrhage NOS \\
\hline & $J 68 z 100$ & Intestinal haemorrhage NOS \\
\hline & $J 68 z 200$ & Upper gastrointestinal haemorrhage \\
\hline & $\mathrm{J} 68 \mathrm{zz} 00$ & $\begin{array}{l}\text { Gastrointestinal tract haemorrhage } \\
\text { NOS }\end{array}$ \\
\hline
\end{tabular}




\section{Authors' affiliations}

T M MacDonald, Medicines Monitoring Unit, Department of Clinical Pharmacology and Therapeutics, University of Dundee, Ninewells Hospital and Medical School, Dundee, UK

S V Morant, Medicines Monitoring Unit, Department of Clinical Pharmacology and Therapeutics, University of Dundee, Ninewells Hospital and Medical School, Dundee, UK, and Cygnus Biostatistics Ltd, Haddenham, UK

J L Goldstein, Department of Medicine, the University of Illinois at Chicago, USA

T A Burke, Pharmacia, Skokie, USA

D Pettitt, Pfizer Inc, New York, USA

Conflict of interest: Professor MacDonald has given scientific advisory board consultancy services on NSAIDs and other therepeutic areas to Pharmacia (now Pfizer) and Pfizer, manufacturers of NSAIDs and COX2 inhibitors. His department has received research grants and research fellow funding from both companies. He has also spoken at events unrelated to NSAIDs for Boehringer Ingelheim, manufacturers of meloxicam. Dr Morant was employed by Searle until 2000, holds stock options in Pharmacia (now Pfizer), and was paid a consultancy fee by Pfizer to conduct this analysis. All three companies market celecoxib.

\section{REFERENCES}

1 Yocum D, Fleischmann R, Dalgin P, et al. Safety and efficacy of meloxicam in the treatment of osteoarthritis: a 12-week, double-blind, multiple-dose, placebo-controlled trial. The Meloxicam Osteoarthritis Investigators. Arch Intern Med 2000; 160:2947-54

2 Martin RM, Biswas P, Mann RD. The incidence of adverse events and risk factors for upper gastrointestinal disorders associated with meloxicam use amongst 19087 patients in general practice in England cohort study. Br J Clin Pharmacol 2000;50:35-42

3 Lanes SF, Garcia Rodriguez LA, Hwang GE. Baseline risk of gastrointestinal disorders among new users of meloxicam, ibuprofen, diclofenac, naproxen and indomethacin. Pharmacoepidemiol Drug Saf 2000;9:1 13-7.

4 Bensen WG, Zhao SZ, Burke TA, et al. Upper gastrointestinal tolerability of celecoxib, a COX-2 specific inhibitor, compared to naproxen and placebo. J Rheumatol 2000;27:1876-83.

5 Bombardier C, Laine L, Reicin A, et al. Comparison of upper gastrointestinal toxicity of rofecoxib and naproxen in patients with rheumatoid arthritis. VIGOR Study Group. N Engl J Med 2000;343:1520-8.
6 Goldstein JL, Silverstein FE, Agrawal NM, et al. Reduced risk of upper gastrointestinal ulcer complications with celecoxib, a novel COX-2 inhibitor. Am J Gastroenterol 2000;95: 1681-90.

7 Langman MJ, Jensen DM, Watson DJ, et al. Adverse upper gastrointestinal effects of rofecoxib compared with NSAIDs. JAMA 1999:282:1929-33.

8 Watson DJ, Harper SE, Zhao PL, et al. Gastrointestinal tolerability of the selective cyclooxygenase-2 (COX-2) inhibitor rofecoxib compared with nonselective COX-1 and COX-2 inhibitors in osteoarthritis. Arch Intern Med 2000; 160:2998-3003

9 McKenna F, Arguelles L, Burke T, et al. Upper gastrointestinal tolerability of celecoxib compared with diclofenac in the treatment of osteoarthritis and rheumatoid arthritis. Clin Exp Rheumatol 2002;20:35-43.

10 Silverstein FE, Faich G, Goldstein JL, et al. Gastrointestinal toxicity with celecoxib vs nonsteroidal anti-inflammatory drugs for osteoarthritis and rheumatoid arthritis The CLASS study: a randomised controlled trial. JAMA 200:284:1247-55.

11 Garcia Rodriguez LA, Perez Gutthann S. Use of the UK General Practice Research Database for pharmacoepidemiology. Br J Clin Pharmacol 1998;45:19-25.

12 Jick H, Jick SS, Derby LE. Validation of information recorded on general practitioner based computerised data resource in the United Kingdom. BM 1991:302:66-8.

13 Rosenbaum PR, Rubin DB. The central role of the propensity score in observational studies for causal effects. Biometrika 1983;70:41-55.

14 Whitehead A, Whitehead J. A general parametric approach to the meta-analysis of randomized clinical trials. Stat Med 1991;10:166577.

15 MacDonald TM, Morant SV, Robinson GC, et al. Association of upper gastrointestinal toxicity of non-steroidal anti-inflammatory drugs with continued exposure: cohort study. BM 1997;315:1333-7.

16 Deeks JJ, Smith LA, Bradley MD. Efficacy, tolerability, and upper gastrointestinal safety of celecoxib for the treatment of osteoarthritis and rheumatoid arthritis: a systematic review of randomised controlled trials. BMV 2002: 325:619-23.

17 Mamdani M, Rochon PA, Juurlink DN, et al. Observational study of upper gastrointestinal haemorrhage in elderly patients given selective cyclo-oxygenase- 2 inhibitors or conventional anti-inflammatory drugs. BM 2002; 325:624-27.

18 Pettitt D, Singh G, Schwartz JS, et al. Determinants of cyclooxygenase (COX)-2 specific inhibitor utilization in a managed care population $\mathrm{Am} J$ Gastroenterol 2000;95:2637 (abstract No 778).

19 Beardon PH, McGilchrist MM, McKendrick AD, et al. Primary non-compliance with prescribed medication in primary care. BM 1993:307:846-8. 